\title{
Lie symmetry of a class of nonlinear boundary value problems with free boundaries
}

\author{
Roman Cherniha ${ }^{\dagger}$ and Sergii Kovalenko ${ }^{\dagger}$ \\ $\dagger$ Institute of Mathematics, Ukrainian National Academy of Sciences, \\ 3 Tereshchenkivs'ka Street, Kyiv 01601, Ukraine \\ E-mail: cherniha@imath.kiev.ua and kovalenko@imath.kiev.ua
}

\begin{abstract}
A class of (1+1)-dimensional nonlinear boundary value problems (BVPs), modeling the process of melting and evaporation of solid materials, is studied by means of the classical Lie symmetry method. New definition of invariance in Lie's sense for BVP is presented and applied for the class of BVPs in question.
\end{abstract}

\section{Introduction}

Boundary value problems of the Stefan type are widely used in mathematical modeling a huge number of processes, which arise in physics, biology, and industry [1], [5], [1], [15], [16]. Nevertheless these processes are very different from formal point of view, they have the common peculiarity, unknown moving boundaries. Movement of unknown boundaries is described by the Stefan boundary conditions. Boundary value problems with the Stefan conditions are the main object of this paper. It is well-known that exact solutions of BVPs of the Stefan type can be derived only in exceptional cases and the relevant list is very short at the present time (see [1], 4], [6], [9], [17] and papers cited therein).

The main idea is to apply the classical Lie symmetry method ( [3], [12]- [14]) to the case of the Stefan type BVPs. The Lie symmetry method is the very powerful tool for investigation of nonlinear partial differential equations (PDEs), notably, for constructing exact solutions. On the other hand, this method is efficient for solving standard BVPs (no moving boundaries) in exceptional cases because the boundary conditions are usually not invariant under any transformations.

Nevertheless the Stefan type problems are more complicated objects than BVPs with fixed boundaries, we have recently noted that the Lie symmetry method should be more applicable just for solving problems with moving boundaries [8]. In fact, the structure of such boundaries may depend on invariant variable(s) and this allows us to reduce the given BVP to one of lower dimensionality. It should be noted that the rigorous definition of Lie invariance for BVPs and relevant examples are presented in [2,3]. However, this definition does not suit to many realistic BVPs of the Stefan type and should be modified and generalized.

Let us consider the following class of the Stefan type BVPs used to describe melting and evaporation of materials in case that their surface is exposed to a powerful flux of energy

$$
\begin{aligned}
& \frac{\partial}{\partial x}\left(\lambda_{1}\left(T_{1}\right) \frac{\partial T_{1}}{\partial x}\right)=\rho_{1}\left(T_{1}\right) c_{1}\left(T_{1}\right) \frac{\partial T_{1}}{\partial t}, \\
& \frac{\partial}{\partial x}\left(\lambda_{2}\left(T_{2}\right) \frac{\partial T_{2}}{\partial x}\right)=\rho_{2}\left(T_{2}\right) c_{2}\left(T_{2}\right) \frac{\partial T_{2}}{\partial t}, \\
& S_{1}(t, x)=0: \lambda_{1}\left(T_{1}\right) \frac{\partial T_{1}}{\partial x}=\rho_{1}\left(T_{1}\right) L_{v} V_{1}-Q\left(t, T_{1}\right), V_{1}=H\left(t, T_{1}\right), \\
& \quad S_{2}(t, x)=0: \lambda_{2}\left(T_{m}\right) \frac{\partial T_{2}}{\partial x}=\lambda_{1}\left(T_{m}\right) \frac{\partial T_{1}}{\partial x}+\rho_{2}\left(T_{m}\right) L_{m} V_{2}, T_{1}=T_{2}=T_{m}, \\
& \quad x=+\infty: T_{2}=T_{0},
\end{aligned}
$$


where $T_{m}, T_{0}$ are the known temperatures of melting and solid phase of material, respectively; $\lambda_{k}\left(T_{k}\right), c_{k}\left(T_{k}\right)$, and $\rho_{k}\left(T_{k}\right)$ are positive thermal conductivities, specific heat, and densities, respectively; $L_{v}, L_{m}$ are latent heats of phase change per unit mass of liquid and solid phases, respectively; $Q\left(t, T_{1}\right)$ is a function that represents the energy flux being absorbed by the metal; $H\left(t, T_{1}\right)$ is a given function; $S_{k}(t, x)$ are the phase division boundary surfaces to be found; $V_{k}(t, x)=-\frac{\partial S_{k}}{\partial t} / \frac{\partial S_{k}}{\partial x}$ are the phase division boundary velocities; $T_{k}(t, x)$ are unknown temperature fields; index $k=1,2$ corresponds to the liquid and solid phases, respectively.

Here Eqs. (11)-(2) are basic and describe the heat transfer process in liquid and solid phases, the boundary conditions (13) present the evaporation dynamics on the surface $S_{1}$, and the boundary conditions (4) are the well-known Stefan conditions on the surface $S_{2}$ dividing the liquid and solid phases. Assuming that the liquid phase thickness is considerably less than the solid phase thickness, one may use the Dirichlet condition (5). It should be stressed that we neglect the initial temperature distribution in solid phase and consider the process on the stage when two phases have already taken place.

One may claim that formulae (1) -(5) present the class of BVPs with moving boundaries and take into account a number of different situations, which occur in the melting and evaporation processes. For example, setting $Q\left(t, T_{1}\right)=$ const, $V_{1}=\operatorname{const} \Phi(t)$ and $H\left(t, T_{1}\right)=\Phi(t) T_{1}$, where $\Phi(t)$ is a correctly-specified function, one obtains the problem, which is the most typical [6], [9]. In the case of the process when surfaces are exposed to very powerful periodic laser impulses these functions take a complicated form [10].

The paper is organized as follows. In section 2, we discuss the existing definition of Lie invariance for BVPs and present its generalization to a wider class of BVPs. In the next section, we apply the definition derived to BVP (11) -(5) . In section 3, all possible Lie operators of the nonlinear system (11)-(2), which allow us to reduce the problem in question to one for ordinary differential equations, are found. Finally, we present conclusions in the last section.

\section{Definition of Lie symmetry for BVPs}

We start from the well-known definition of invariance of a BVP under the given infinitesimal operator presented in [3]. We restrict ourselves to the case when the basic equation of BVP is a two-dimensional evolution PDE of $k$ th-order $(k \geq 2)$. In this case the relevant BVP may be formulated as follows:

$$
\begin{gathered}
u_{t}=F\left(x, u, u_{x}, \ldots, u_{x}^{(k)}\right),(t, x) \in \Omega \subset \mathbf{R}^{2} \\
s_{a}(t, x)=0: B_{a}\left(t, x, u, u_{x}, \ldots, u_{x}^{(k-1)}\right)=0, a=1,2, \ldots, p,
\end{gathered}
$$

where $F$ and $B_{a}$ are smooth functions in the corresponding domains, $\Omega$ is a domain with smooth boundaries and $s_{a}(t, x)$ are smooth curves. Hereafter the subscripts $t$ and $x$ denote differentiation with respect to these variables, $u_{x}^{(j)}=\frac{\partial^{j} u}{\partial x^{j}}, j=1,2, \ldots, k$. We assume that BVP (6) -(7) has a classical solution (in a usual sense).

Consider the infinitesimal generator

$$
X=\xi^{0}(t, x) \frac{\partial}{\partial t}+\xi^{1}(t, x) \frac{\partial}{\partial x}+\eta(t, x, u) \frac{\partial}{\partial u},
$$

(hereafter $\xi^{0}, \xi^{1}$ and $\eta$ are known smooth functions), which defines a Lie symmetry acting on both $(t, x, u)$-space as well as on its projection to $(t, x)$-space. Let $X^{(k)}$ be the $k$ th-prolongation of the generator $X$ calculated by the well-known prolongation formulae (see, e.g. [12]- [14]). 
Definition 1 [3] The Lie symmetry $X$ (8) is admitted by the boundary value problem (6) - (7) if and only if:

(a) $X^{(k)}\left(F\left(x, u, u_{x}, \ldots, u_{x}^{(k)}\right)-u_{t}\right)=0$ when $u$ satisfies (6));

(b) $X\left(s_{a}(t, x)\right)=0$ when $s_{a}(t, x)=0, a=1,2, \ldots, p$;

(c) $X^{(k-1)}\left(B_{a}\left(t, x, u, u_{x}, \ldots, u_{x}^{(k-1)}\right)\right)=0$ when $\left.B_{a}\right|_{s_{a}(t, x)=0}=0, a=1,2, \ldots, p$.

One easily notes that Definition 1 can not be applied to BVP (1)-(5), because, firstly, there are two basic equations (11)-(2) instead of one, secondly, the boundary condition (15) is defined on the non-regular manifold $x=\infty$, thirdly, there are several boundary conditions on the moving surfaces $S_{1}(t, x)$ and $S_{2}(t, x)$. Thus, this definition should be extended to the wider class of BVPs.

Consider a BVP for the $m$-component system of evolution equations with two independent $(t, x)$ and $m$ dependent $u=\left(u_{1}, u_{2}, \ldots, u_{m}\right)$ variables. Let us assume that the $k$ th-order $(k \geq 2)$ basic equations of evolution type

$$
u_{t}^{i}=F^{i}\left(x, u, u_{x}, \ldots, u_{x}^{(k)}\right), i=1,2, \ldots, m,
$$

are defined on a domain $\Omega \subset \mathbf{R}^{2}$ and there are three types of boundary conditions, which can arise in applications:

$$
\begin{gathered}
s_{a}(t, x)=0: B_{a}^{j}\left(t, x, u, u_{x}, \ldots, u_{x}^{\left(k_{j a}\right)}\right)=0, a=1, \ldots, p, j=1, \ldots, n_{a}, \\
S_{b}(t, x)=0: B_{b}^{l}\left(t, x, u, \ldots, u_{x}^{\left(k_{l b}\right)}, S_{b}, \frac{\partial S_{b}}{\partial t}, \frac{\partial S_{b}}{\partial x}\right)=0, b=1, \ldots, q, l=1, \ldots, n_{b},
\end{gathered}
$$

and

$$
\gamma_{c}(t, x)=\infty: \Gamma_{c}\left(t, x, u, u_{x}, \ldots, u_{x}^{\left(k_{c}\right)}\right)=0, c=1,2, \ldots, r .
$$

Here $k_{j a}<k$ and $k_{l b}<k$ are the given numbers, $s_{a}(t, x)$ and $\gamma_{c}(t, x)$ are the known functions, while the functions $S_{b}(t, x)$ defining free boundary surfaces must be found. We assume that all functions arising in (9)-(12) are sufficiently smooth so that a classical solution exists for this BVP.

Consider the infinitesimal generator

$$
X=\xi^{0}(t, x) \frac{\partial}{\partial t}+\xi^{1}(t, x) \frac{\partial}{\partial x}+\eta^{1}(t, x, u) \frac{\partial}{\partial u^{1}}+\ldots+\eta^{m}(t, x, u) \frac{\partial}{\partial u^{m}},
$$

which defines a Lie symmetry acting on both $(t, x, u, S)$-space (here the notation $S=$ $\left(S_{1}, S_{2}, \ldots, S_{q}\right)$ is used) as well as on its projection to $(t, x)$-space and generates a Lie group of the point transformations

$$
t^{\prime}=T(t, x, \varepsilon), x^{\prime}=X(t, x, \varepsilon), u_{j}^{\prime}=U_{j}(t, x, u, \varepsilon), S_{b}^{\prime}=S_{b}(t, x) .
$$

Definition 2 BVP (9)-(12) admits the one-parameter Lie group of transformations (14) generated by the infinitesimal operator (13) if and only if:

(a) $X^{(k)}\left(F^{i}\left(x, u, u_{x}, \ldots, u_{x}^{(k)}\right)-u_{t}^{i}\right)=0$ when the functions $u_{i}, i=1,2, \ldots, m$ satisfy (9) ;

(b) $X\left(s_{a}(t, x)\right)=0$ when $s_{a}(t, x)=0, \quad a=1,2, \ldots, p$; 
(c) $X^{\left(k_{j a}\right)}\left(B_{a}^{j}\left(t, x, u, u_{x}, \ldots, u_{x}^{\left(k_{j a}\right)}\right)\right)=0$ when $\left.B_{a}^{j}\right|_{s_{a}(t, x)=0}=0, \quad a=1,2, \ldots, p, j=$ $1, \ldots, n_{a}$;

(d) $X^{\left(k_{l b}\right)}\left(B_{b}^{l}\left(t, x, u, \ldots, u_{x}^{\left(k_{l b}\right)}, S_{b}, \frac{\partial S_{b}}{\partial t}, \frac{\partial S_{b}}{\partial x}\right)\right)=0$ when $\left.B_{b}^{l}\right|_{S_{b}(t, x)=0}=0, b=1, \ldots, q, l=$ $1, \ldots, n_{b}$

(e) $X_{*}\left(\gamma_{c}^{*}(\tau, y)\right)=0$ when $\gamma_{c}^{*}(\tau, y)=0, c=1,2, \ldots, r$;

(f) $X_{*}^{\left(k_{c}\right)}\left(\Gamma_{c}^{*}\left(\tau, y, u, u_{y}, \ldots, u_{y}^{\left(k_{c}\right)}\right)\right)=0$ when $\left.\Gamma_{c}^{*}\right|_{\gamma_{c}^{*}(\tau, y)=0}=0, \quad c=1,2, \ldots, r$,

where $X_{*}, \Gamma_{b}^{*}$ and $\gamma_{c}^{*}(\tau, y)$ are operator (13), the functions $\Gamma_{b}$ and $\frac{1}{\gamma_{c}(t, x)}$, respectively, expressed via the new independent variables $\tau=\left\{\begin{array}{ll}t, & \text { if } \frac{\partial \gamma_{c}(t, x)}{\partial x} \neq 0, \\ \frac{1}{\gamma_{c}(t, x)}, & \text { if } \frac{\partial \gamma_{c}(t, x)}{\partial x}=0\end{array}\right.$ and $y=$

$\begin{cases}x, & \text { if } \quad \frac{\partial \gamma_{c}(t, x)}{\partial x}=0 \\ \frac{1}{\gamma_{c}(t, x)}, & \text { if } \frac{\partial \gamma_{c}(t, x)}{\partial x} \neq 0 .\end{cases}$

It should be noted that this Definition coincides with Definition 1 if $m=1, n_{a}=1$ and there are no boundary conditions of the form (11) and (12). On the other hand, the following example shows that the generalization of Definition 1 presented above is non-trivial.

Example. Let us consider BVP (9) -(10), which includes also the boundary conditions

$$
x=\infty: \Gamma_{c}(u)=0, c=1,2, \ldots, r .
$$

Assume that system (9) and conditions (10) are invariant under the group of translations on the plane $(t, x)$ :

$$
t^{\prime}=t+\lambda_{1} \varepsilon, \quad x^{\prime}=x+\lambda_{2} \varepsilon, \quad u^{\prime}=u, \quad \lambda_{1} \lambda_{2} \neq 0,
$$

so that the corresponding infinitesimal generator

$$
X=\lambda_{1} \partial_{t}+\lambda_{2} \partial_{x}
$$

satisfies items (a)-(c) from Definition 2. Moreover, according to Definition 2 the operator $X$ of the form (16) and the functions $\Gamma_{c}(u)$ and $\gamma_{c}(t, x)=x$ take the form

$$
X_{*}=\lambda_{1} \partial_{\tau}-\lambda_{2} y^{2} \partial_{y}
$$

$\Gamma_{c}^{*}(u)=\Gamma_{c}(u)$ and $\gamma_{c}^{*}(\tau, y)=y$, respectively. Now one easily checks that items (e) and (f) from Definition 2 are satisfied. Thus, BVP in question admits the Lie group of point transformations (15) generated by the infinitesimal generator (16).

However, the problem with non-regular manifold $\gamma_{c}(t, x)=\infty$ occurs if one generalizes Definition 1 in the standard way and formulates items (e) and (f) from Definition 2 like those (b) and (c). For example, this manifold can not be replaced by the regular one $\gamma_{c}(t, x)-L=0$, where $L \rightarrow \infty$ because this leads to the requirement

$$
\left.\lim _{L \rightarrow \infty} X\left(\gamma_{c}(t, x)-L\right)\right|_{\gamma_{c}(t, x)=L}=0
$$

On the other hand, this requirement is not satisfied in the example presented above because

$$
\left.\lim _{L \rightarrow \infty} X(x-L)\right|_{x=L}=\lim _{L \rightarrow \infty} \lambda_{2}=\lambda_{2} \neq 0 .
$$

Thus, item (e) in Definition 2 can not be formulated like item (b).

Hereafter Definition 2 will be applied to derive the invariance operators of BVP (11)-(5). 


\section{Invariance of BVP (1)-(5) under the Lie generators}

It should be noted that BVP (1) -(5) can be simplified if one applies the Goodman substitution

$$
u=\phi_{1}\left(T_{1}\right) \equiv \int_{0}^{T_{1}} c_{1}(\zeta) \rho_{1}(\zeta) d \zeta, \quad v=\phi_{2}\left(T_{2}\right) \equiv \int_{0}^{T_{2}} c_{2}(\xi) \rho_{2}(\xi) d \xi .
$$

Substituting (17) into (11)-(5) and making the relevant calculations, we arrive at the equivalent BVP of the form

$$
\begin{aligned}
\frac{\partial u}{\partial t}= & \frac{\partial}{\partial x}\left(d_{1}(u) \frac{\partial u}{\partial x}\right) \\
\frac{\partial v}{\partial t}= & \frac{\partial}{\partial x}\left(d_{2}(v) \frac{\partial v}{\partial x}\right) \\
& S_{1}(t, x)=0: d_{1}(u) \frac{\partial u}{\partial x}=\hat{\rho}_{1}(u) L_{v} V_{1}-\hat{q}(t, u), V_{1}=\hat{h}(t, u), \\
& S_{2}(t, x)=0: d_{2}\left(v_{m}\right) \frac{\partial v}{\partial x}=d_{1}\left(u_{m}\right) \frac{\partial u}{\partial x}+\hat{\rho}_{2}\left(v_{m}\right) L_{m} V_{2}, u=u_{m}, v=v_{m}, \\
& x=+\infty: v=v_{\infty},
\end{aligned}
$$

where $u_{m}=\int_{0}^{T_{m}} c_{1}(\zeta) \rho_{1}(\zeta) d \zeta, \quad v_{m}=\int_{0}^{T_{m}} c_{2}(\xi) \rho_{2}(\xi) d \xi, \quad v_{\infty}=\int_{0}^{T_{0}} c_{2}(\xi) \rho_{2}(\xi) d \xi ; \quad d_{1}(u)=$ $\frac{\lambda_{1}\left(\phi_{1}^{-1}(u)\right)}{c_{1}\left(\phi_{1}^{-1}(u)\right) \rho_{1}\left(\phi_{1}^{-1}(u)\right)}, d_{2}(v)=\frac{\lambda_{2}\left(\phi_{2}^{-1}(v)\right)}{c_{2}\left(\phi_{2}^{-1}(v)\right) \rho_{2}\left(\phi_{2}^{-1}(v)\right)} ; \hat{\rho}_{1}(u)=\rho_{1}\left(\phi_{1}^{-1}(u)\right), \hat{\rho}_{2}(v)=\rho_{2}\left(\phi_{2}^{-1}(v)\right), \hat{q}(t, u)=$ $Q\left(t, \phi_{1}^{-1}(u)\right)$, and $\hat{h}(t, u)=H\left(t, \phi_{1}^{-1}(u)\right)$ (here $\phi_{i}^{-1}$ are inverse functions to $\phi_{i}$, the functions $d_{1}(u)$ and $d_{2}(v)$ are strictly positive and $\left.v_{m} \neq v_{\infty}\right)$.

Now one sees that BVP (18)-(22) is based on the standard nonlinear heat equations (NHE). Lie symmetries of the non-coupled system (18)-(19) can be easily derived from paper [7], where reaction-diffusion systems of more general form have been investigated. The result is presented in Table 1 (note, we do not consider the case when this system is linear). Using Definition 2 and Lie symmetries from Table 1 the following theorem can be established.

Theorem 1 A nonlinear BVP of the form (18)-(22) admits a Lie symmetry operator of system (18)-(19) if and only if the operator in question up to the local transformations $x \rightarrow x+x_{0}, t \rightarrow$ $t+t_{0}\left(x_{0} \in \mathbf{R}, t_{0} \in \mathbf{R}\right)$ is equivalent either to the operator

$$
X_{1}=\partial_{t}+\mu \partial_{x}, \mu \in \mathbf{R}
$$

or to

$$
X_{2}=2 t \partial_{t}+x \partial_{x} .
$$

Moreover, the functions $\hat{f}(t, u), \hat{q}(t, u)$ must have the correctly specified forms

$$
\hat{h}(t, u)=h(u), \hat{q}(t, u)=q(u)
$$

and

$$
\hat{h}(t, u)=\frac{h(u)}{\sqrt{t}}, \hat{q}(t, u)=\frac{q(u)}{\sqrt{t}},
$$

respectively. Here $q(u)$ and $h(u)$ are arbitrary smooth functions. 
Proof. Firstly, we derive the group classification of the NHE system (18)-(19) using the determining equations presented in [7]. If $d_{1}(u)$ and $d_{2}(v)$ are arbitrary functions then those equations immediately give the three-dimensional maximal algebra of invariance (MAI) $A=$ $\left\langle\partial_{t}, \partial_{x}, 2 t \partial_{t}+x \partial_{x}\right\rangle$ called the principal algebra. There are five special cases when an extension of the principal algebra occurs and they are listed in Table 1.

It should be stressed that each NHE system admitting four- or five-dimensional Lie algebra is reduced to one of those with diffusivities from Table 1 by the equivalence transformations

$$
t \rightarrow e_{0} t+t_{0}, \quad x \rightarrow e_{1} x+x_{0}, \quad u \rightarrow e_{2} u+u_{0}, \quad v \rightarrow e_{3} v+v_{0},
$$

where $e_{i} \neq 0(i=0, \ldots, 3), t_{0}, x_{0}, u_{0}$, and $v_{0}$ are arbitrary parameters. It turns out that the class of BVPs (18)-(22) is also invariant under transformations (27) and this can be easily checked by direct calculations.

Thus, on the next stage, we need to apply Definition 2 only to BVP (18)-(22) in six cases listed in Table 1.

Table 1. Lie algebras of the NHE system (18)-(19).

\begin{tabular}{|c|c|c|c|}
\hline$n o$ & $d_{1}(u)$ & $d_{2}(v)$ & Basic operators of MAI \\
\hline \hline 1. & $\forall$ & $\forall$ & $A=\left\langle\partial_{t}, \partial_{x}, 2 t \partial_{t}+x \partial_{x}\right\rangle$ \\
2. & $\forall$ & 1 & $A, v \partial_{v}, \beta(t, x) \partial_{v}$ \\
3. & $e^{u}$ & $e^{v}$ & $A, x \partial_{x}+2 \partial_{u}+2 \partial_{v}$ \\
4. & $e^{u}$ & $v^{m}$ & $A, x \partial_{x}+2 \partial_{u}+\frac{2}{m} v \partial_{v}$ \\
5. & $u^{n}$ & $v^{m}$ & $A, x \partial_{x}+\frac{2}{n} u \partial_{u}+\frac{2}{m} v \partial_{v}$ \\
6. & $u^{-\frac{4}{3}}$ & $v^{-\frac{4}{3}}$ & $A, x \partial_{x}-\frac{3}{2} u \partial_{u}-\frac{3}{2} v \partial_{v}, x^{2} \partial_{x}-3 x u \partial_{u}-3 x v \partial_{v}$ \\
\hline
\end{tabular}

Let us consider the first case when the functions $d_{1}(u)$ and $d_{2}(v)$ are arbitrary. In this case the most general form of the Lie symmetry generator is

$$
X=\left(\lambda_{1}+2 \lambda_{3} t\right) \partial_{t}+\left(\lambda_{2}+\lambda_{3} x\right) \partial_{x}
$$

(hereinafter $\lambda$ with indices are arbitrary constants). Obviously, operator (28) can be reduced either to the form (23) (if $\lambda_{3}=0$ and $\lambda_{1} \neq 0$ ) or to (24) (if $\lambda_{3} \neq 0$ ) by the local transformations

$$
x \rightarrow x+x_{0}, t \rightarrow t+t_{0}\left(x_{0} \in \mathbf{R}, t_{0} \in \mathbf{R}\right),
$$

which belong to (27).

Note that the case $\lambda_{3}=\lambda_{1}=0$ leads to the invariance operator $X=\partial_{x}$. However, this operator generates the Lie ansatz, which does not alow existing the moving boundary surfaces $S_{k}(t, x), k=1,2$, hence, we do not consider this non-physical case.

Let us apply Definition 2 to prove the invariance of BVP (18)-(22) under the Lie symmetry operator (23). Applying the first prolongation of generator (23),$X_{1}^{(1)}=X_{1}$, to the boundary conditions (20), we obtain

$$
\begin{aligned}
& \left.X_{1}\left(d_{1}(u) \frac{\partial u}{\partial x}-\hat{\rho}_{1}(u) L_{v} V_{1}+\hat{q}(t, u)\right)\right|_{\mathcal{M}}=\frac{\partial \hat{q}(t, u)}{\partial t}, \\
& \left.X_{1}\left(V_{1}-\hat{h}(t, u)\right)\right|_{\mathcal{N}}=-\frac{\partial \hat{h}(t, u)}{\partial t} .
\end{aligned}
$$


where the manifold $\mathcal{M}=\left\{S_{1}(t, x)=0: d_{1}(u) \frac{\partial u}{\partial x}=\hat{\rho}_{1}(u) L_{v} V_{1}-\hat{q}(t, u)\right\}$ and the manifold $\mathcal{N}=$ $\left\{S_{1}(t, x)=0: V_{1}=\hat{h}(t, u)\right\}$. According to item (d) from Definition 2 we arrive at the restriction on the function $\hat{q}(t, u)$ and $\hat{h}(t, u)$

$$
\begin{gathered}
\frac{\partial \hat{q}(t, u)}{\partial t}=0 \Leftrightarrow \hat{q}=q(u), \\
\frac{\partial \hat{h}(t, u)}{\partial t}=0 \Leftrightarrow \hat{h}=h(u) .
\end{gathered}
$$

The invariance of conditions (21) are trivially fulfilled because they do not involve the variables $t$ and $x$ in explicit form. Finally, condition (22) satisfies items (e) and (f) from Definition 2 because this immediately follows from Example.

The invariance of BVP (18)-(22) under generator (24) is checked in a similar way. Note that the first prolongation of generator (24) has the more complicated form than one of (23) , namely

$$
X_{2}^{(1)}=2 t \partial_{t}+x \partial_{x}-2 u_{t} \partial_{u_{t}}-2 v_{t} \partial_{v_{t}}-u_{x} \partial_{u_{x}}-v_{x} \partial_{v_{x}}-2 S_{t}^{1} \partial_{S_{t}^{1}}-2 S_{t}^{2} \partial_{S_{t}^{2}}-S_{x}^{1} \partial_{S_{x}^{1}}-S_{x}^{2} \partial_{S_{x}^{2}}
$$

Since the boundary conditions (20) involve the first-order derivatives (we remind that $V_{k}=$ $\left.-\frac{\partial S_{k}}{\partial t} / \frac{\partial S_{k}}{\partial x}\right)$ we have calculated how operator (29) acts on these boundary conditions according to Definition 2 and obtained two ODEs of the form

$$
2 t \frac{\partial \hat{q}(t, u)}{\partial t}+\hat{q}(t, u)=0, \quad 2 t \frac{\partial \hat{h}(t, u)}{\partial t}+\hat{h}(t, u)=0,
$$

which lead to the restrictions (26).

Thus, the case of arbitrary functions $d_{1}(u)$ and $d_{2}(v)$ is completely examined.

It turns out that any other Lie generator arising in Table 1 does not satisfy Definition 2 for BVP (18)-(22). Let us consider, for instance, case 4 of Table 1 . Here, the most general form of the Lie symmetry generator is

$$
X_{3}=\left(\lambda_{1}+2 \lambda_{3} t\right) \partial_{t}+\left(\lambda_{2}+\left(\lambda_{3}+\lambda_{4}\right) x\right) \partial_{x}+2 \lambda_{4} \partial_{u}+\frac{2}{m} \lambda_{4} v \partial_{v}
$$

where we assume $\lambda_{4} \neq 0$, otherwise $X_{3}$ takes form (28).

In order to be invariant under generator (30), the second condition from (21) must satisfy item (d) from Definition 2

$$
\left.X_{3}\left(u-u_{m}\right)\right|_{\mathcal{P}}=0,
$$

where $\mathcal{P}=\left\{S_{2}(t, x)=0: u=u_{m}\right\}$, however, this leads to the restriction $\lambda_{4}=0$. Thus, the contradiction is obtained and we conclude that generator (30) is not Lie symmetry of any BVP (18) -(22) with diffusivities from case 4 of Table 1.

In a similar way, cases 2,3,5 and 6 listed in Table 1 have been examined and contradictions with boundary value conditions established.

The proof is now complete.

Remark 1 The group classification of the NHE system (18)-(19) presented in Table 1 takes into account the trivial discrete transformations $u \rightarrow v, v \rightarrow u$ admitted by this system. Nevertheless, the class of BVPs (18)-(22) is not invariant under these transformations (see conditions (20)) it does not affect Theorem 1. 
Using Theorem 1 one can reduce the class of BVPs (18)-(22) to two classes of BVPs for second-order ODEs. In fact, substituting ansätze

$$
\begin{aligned}
& u=u(\xi), \quad v=v(\xi), \quad S_{k}=S_{k}(\xi), \quad \xi=x-\mu t \\
& u=u(\omega), \quad v=v(\omega), \quad S_{k}=S_{k}(\omega), \quad \omega=\frac{x}{\sqrt{t}}
\end{aligned}
$$

generated by operators (23) and (24), respectively, and taking into account restrictions (25) and (26) the following consequences are obtained.

Corollary 1 Ansatz (31) reduces a nonlinear BVP of the form (18)-(22) with the coefficient restrictions (25) to the following BVP for the second-order ODEs

$$
\begin{aligned}
& \frac{d}{d \xi}\left(d_{1}(u) \frac{d u}{d \xi}\right)+\mu \frac{d u}{d \xi}=0, \quad 0<\xi<\delta \\
& \frac{d}{d \xi}\left(d_{2}(v) \frac{d v}{d \xi}\right)+\mu \frac{d v}{d \xi}=0, \quad \xi>\delta \\
& \xi=0: d_{1}(u) \frac{d u}{d \xi}=\hat{\rho}_{1}(u) L_{v} \mu-q(u), \mu=h(u), \\
& \xi=\delta: d_{2}\left(v_{m}\right) \frac{d v}{d \xi}=d_{1}\left(u_{m}\right) \frac{d u}{d \xi}+\hat{\rho}_{2}\left(v_{m}\right) L_{m} \mu, u=u_{m}, v=v_{m} \\
& \xi=+\infty: v=v_{\infty},
\end{aligned}
$$

where $\delta$ and $\mu$ are parameters to be found.

Corollary 2 Ansatz (32) reduces a nonlinear BVP of the form (18)-(20) with the coefficient restrictions (26) to the following BVP for the second-order ODEs

$$
\begin{aligned}
& \frac{d}{d \omega}\left(d_{1}(u) \frac{d u}{d \omega}\right)+\frac{\omega}{2} \frac{d u}{d \omega}=0, \quad \omega_{1}<\omega<\omega_{2}, \\
& \frac{d}{d \omega}\left(d_{2}(v) \frac{d v}{d \omega}\right)+\frac{\omega}{2} \frac{d v}{d \omega}=0, \quad \omega>\omega_{2}, \\
& \omega=\omega_{1}: d_{1}(u) \frac{d u}{d \omega}=\hat{\rho}_{1}(u) L_{v} \frac{\omega_{1}}{2}-q(u), \frac{\omega_{1}}{2}=h(u), \\
& \omega=\omega_{2}: d_{2}\left(v_{m}\right) \frac{d v}{d \omega}=d_{1}\left(u_{m}\right) \frac{d u}{d \omega}+\hat{\rho}_{2}\left(v_{m}\right) L_{m} \frac{\omega_{2}}{2}, u=u_{m}, v=v_{m}, \\
& \omega=+\infty: v=v_{\infty},
\end{aligned}
$$

where $\omega_{1}$ and $\omega_{2}$ are parameters to be found.

It should be noted that each BVP (33)-(37) with the correctly specified functions $q(u)$ and $h(u)$ arising in (35) can exactly be solved and an exact solution can be presented at least in an implicit form (see for details [6]). Some BVPs (38)-(42) with the correctly specified coefficients arising in the problem were exactly solved in particular cases [8].

\section{Conclusions}

In this paper, the class of $(1+1)$-dimensional nonlinear boundary value problems (1)-(5), modeling the process of melting and evaporation of metals, is studied by means of the classical 
Lie symmetry method. New definition of invariance in Lie's sense is presented. This definition is applicable to wide classes of BVPs, including those with several basic equations, with moving boundaries, and with boundary conditions on non-regular manifolds.

Theorem 1 giving all possible operators of Lie's invariance for the class of BVPs (11)-(15) is proved and the corresponding corollaries, which allow us to reduce the problems in question to those for ODE systems, are also obtained.

Finally, we note that Theorem 2 from the recent paper [8] follows as a particular case from Theorem 1, nevertheless Definition 2 was not used in [8] because the direct application of Lie point transformations was applied therein. This means that definition of Lie's invariance for BVPs can be derived in different ways. We are going to discuss this in details in a forthcoming paper.

\section{References}

[1] V. Alexiades and A. D. Solomon, Mathematical Modeling of Melting and Freezing Processes, Hemisphere Publishing Corporation, Washington, 1993.

[2] G. W. Bluman and S. C. Anco, Symmetry and Integration Methods for Differential Equations, Springer, New York, 2002.

[3] G. W. Bluman and S. Kumei, Symmetries and Differential Equations, Springer-Verlag, New York, 1989.

[4] A. C. Briozzo and D. A. Tarzia, An explicit solution for an instantaneous two-phase Stefan problem with nonlinear thermal coefficients, IMA J. Appl. Math. 67 (2002), 249-261.

[5] N. Britton, Essential Mathematical Biology, Springer, Berlin, 2003.

[6] R. M. Cherniha and N. D. Cherniha, Exact solutions of a class of nonlinear boundary value problems with moving boundaries, J. Phys. A: Math. Gen. 26 (1993), L935-L940.

[7] R. Cherniha and J. R. King, Nonlinear reaction-diffusion systems with variable diffusivities: Lie symmetries, ansätze and exact solutions, J. Math. Anal. Appl. 308 (2005), 11-35.

[8] R. Cherniha and S. Kovalenko, Exact solutions of nonlinear boundary value problems of the Stefan type, J. Phys. A: Math. and Theor. 42 (2009), 355202.

[9] R. M. Cherniha and I. G. Odnorozhenko, Exact solutions of a nonlinear boundary value problem of melting and evaporation of metals under the action of high energy flux, Dopovidi Akad. Nauk Ukrainy (Reports of Acad. Sci. of Ukraine), ser.A 12 (1990), 44-47 (in Ukrainian, Summary in English).

[10] R. M. Cherniha and I. G. Odnorozhenko, Studies of the processes of melting and evaporation of metals under the action of laser radiation pulses, Promyshlennaya Teplotekhnika (Industrial Heat Technic) 13 (1991), 51-59 (in Russian, Summary in English).

[11] J. Crank, Free and Moving Boundary Problems, Clarendon Press, Oxford, 1984.

[12] W. I. Fushchych, W. M. Shtelen and M. I. Serov, Symmetry Analysis and Exact Solutions of Equations of Nonlinear Mathematical Physics, Kluwer, Dordrecht, 1993. 
[13] P. J. Olver, Applications of Lie Groups to Differential Equations, Springer, Berlin, 1986.

[14] L. V. Ovsiannikov, The Group Analysis of Differential Equations, Academic Press, New York, 1982.

[15] J. Ready, Effects of High-Power Laser Radiation, Academic Press, New York, 1971.

[16] L. I. Rubinstein, The Stefan Problem, American Mathematical Society, Providence, 1971.

[17] V. R. Voller, J. B. Swenson and C. Paola, An analytical solution for a Stefan problem with variable latent heat, Int. J. Heat Mass Transfer 47 (2004), 5387-5390. 